\title{
openheart Association of left anterior descending artery involvement on clinical outcomes among patients with STEMI presenting with and without out-of-hospital cardiac arrest
}

Mia Bertic (D) , ${ }^{1}$ Christopher B Fordyce, ${ }^{1}$ Nima Moghaddam (D) , ${ }^{1}$ John Cairns, ${ }^{1}$ Martha Mackay (D) , ${ }^{2}$ Joel Singer, ${ }^{3}$ Terry Lee, ${ }^{3}$ Michele Perry-Arnesen, ${ }^{4}$ Wendy Tocher, ${ }^{4}$ Graham Wong ${ }^{1}$

To cite: Bertic M, Fordyce CB, Moghaddam N, et al. Association of left anterior descending artery involvement on clinical outcomes among patients with STEMI presenting with and without out-of-hospital cardiac arrest. Open Heart 2020;7:e001065. doi:10.1136/ openhrt-2019-001065

Received 16 April 2019 Revised 13 December 2019 Accepted 5 January 2020

Check for updates

(c) Author(s) (or their employer(s)) 2020. Re-use permitted under CC BY-NC. No commercial re-use. See rights and permissions. Published by BMJ.

${ }^{1}$ Division of Cardiology, University of British Columbia, Vancouver, British Columbia, Canada

${ }^{2}$ Department of Medicine, Providence Health Care, Vancouver, British Columbia, Canada

${ }^{3}$ Centre for Health Evaluation and Outcome Sciences, St. Paul's Hospital and University of British Columbia, Vancouver, British Columbia, Canada ${ }^{4}$ Vancouver Coastal Health Authority, Vancouver, British Columbia, Canada

Correspondence to Dr Graham Wong; gcwong@ mail.ubc.ca

\section{ABSTRACT}

Background ST-segment elevation myocardial infarction (STEMI) outcomes are influenced by the location of the culprit vessel with worse outcomes portended with a left anterior descending (LAD) culprit lesion. However, relatively little is known about the independent association of LAD involvement with clinical outcomes of patients with STEMI with and without outof-hospital cardiac arrest (OHCA).

Methods We identified 91 patients with and 929 without a preceding OHCA within the Vancouver Coastal Health Authority who presented with an acute STEMI and underwent primary percutaneous coronary intervention between 26 June 2007 and 31 March 2016.

Results Patients with STEMI with OHCA had higher rates of in-hospital cardiac arrest $(43.3 \%$ vs $8.3 \%, p<0.001)$, heart failure $(50.5 \%$ vs $11.3 \%, \mathrm{p}<0.001)$, cardiogenic shock $(49.5 \%$ vs $5.7 \%, p<0.001)$, mortality $(35.2 \%$ vs $3.3 \%, p<0.001)$ and reduced left ventricular ejection fraction (LVEF; $42.9 \%$ vs $47.3 \%, p<0.001$ ) compared with those without OHCA. Among patients without OHCA, LAD involvement was associated with increased heart failure $(18.1 \%$ vs $5.2 \%, p<0.001)$, in-hospital cardiac arrest $(10.7 \%$ vs $6.2 \%, p<0.014)$, cardiogenic shock $(8.4 \%$ vs $3.3 \%, p<0.001)$, reduced LVEF $(43.0 \%$ vs $51.2 \%, p<0.001)$ and mortality $(5.2 \%$ vs $1.3 \%, p=0.003)$ compared with patients without LAD involvement. With the exception of LVEF, these associations were not seen among patients with STEMI with OHCA and an LAD culprit. The presence of an LAD culprit was not independently associated with increased hospital mortality among patients with OHCA after adjusting for potential confounding factors.

Conclusion Our study has demonstrated a differential impact of LAD involvement on clinical outcomes among patients with STEMI who present with and without OHCA. Our data highlight the complexity surrounding the prognostication following OHCA complicating STEMI and demonstrate that other mechanisms other than LAD involvement contribute to the high mortality associated with OHCA as a result of STEMI.

\section{Key questions}

What is already known about this subject?

- There are conflicting data with smaller studies examining the impact of angiographic features on outcomes in patients with out-of-hospital cardiac arrest (OHCA). Tofler et al found that there were no differences in long-term mortality of patients presenting with ventricular tachycardia or ventricular fibrillation (VT/VF) compared with those without VT/VF when they were separated into those with anterior STsegment elevation myocardial infarction (STEMI) or inferior or posterior STEMI. In contrast, a multicentre analysis by Velders et al with 224 patients with STEMI with OHCA described an association between culprit artery and $\mathrm{OHCA}$, with the risk being highest for proximal left coronary lesions.

What does this study add?

- Prior to our study, little was known about whether angiographic features (and, in particular, left anterior descending (LAD) involvement) independently conferred higher in-hospital mortality and worse inhospital outcomes among patients with STEMI with OHCA. Our study is one of the largest studies using contemporary STEMI data evaluating the association of LAD on outcomes among patients with STEMI with and without OHCA. Our data demonstrated that there was no independent association of LAD with mortality among patients with STEMI presenting with OHCA.

\section{BACKGROUND}

Out-of-hospital cardiac arrest (OHCA) remains a significant public health issue associated with high mortality ${ }^{1}{ }^{2}$ with an overall survival of only $10 \%$ to hospital discharge. ${ }^{34}$ An acute coronary syndrome is a frequent cause of OHCA and rapid access to emergent primary percutaneous coronary intervention (PCI) in OHCA caused 


\section{Key questions}

How might this impact on clinical practice?

- Our study highlights the complexity surrounding the prognostication following OHCA while suggesting that mechanisms other than $\mathrm{LAD}$ involvement confers a higher mortality and worse hospital outcomes in patients with OHCA following successfully reperfusion with primary percutaneous coronary intervention. Clinicians treating patients with STEMI and OHCA should continue to use management strategies that emphasise early identification of patients with STEMI with OHCA and rapid reperfusion but should also focus on identifying and managing other concomitant high-risk features driving poor outcomes in these patients, such as shock and neurological injury.

by ST-segment elevation myocardial infarction (STEMI) is associated with improved survival. ${ }^{5-7}$ Current North American and European cardiac arrest and resuscitation guidelines indicate a strong recommendation for angiography and PCI in patients with OHCA with STEMI. ${ }^{89}$

Predictors of poor outcomes in patients with OHCA with STEMI include increased age, delayed cardiopulmonary resuscitation (CPR), initial asystole rhythm, renal insufficiency, delayed time to return of spontaneous circulation (ROSC) and reduced ejection fraction on hospital admission. ${ }^{10}$ The location of the culprit vessel is also an important clinical predictor of outcomes in STEMI. STEMI due to a left anterior descending (LAD) culprit vessel is associated with worse clinical outcomes due to the extent of myocardial involvement. ${ }^{11-13} \mathrm{An}$ anterior location of a myocardial infarction is an important independent predictor of mortality among patients with STEMI undergoing primary PCI (PPCI). ${ }^{11} 14$ However, little is known about the independent impact of coronary territory involvement, in particular the LAD, on outcomes acute STEMI associated with OHCA. ${ }^{15-19}$

Our objective was to describe the characteristics of patients with STEMI who presented with and without OHCA, and to describe any differences in the distribution of an LAD culprit vessel among patients with STEMI with and without OHCA. We also aimed to compare the in-hospital outcomes in patients with STEMI with and without OHCA and outcomes of patients with STEMI with and without OHCA based on the presence of an LAD culprit. The final aim of this study was to determine if there was any independent association between an LAD culprit vessel and in-hospital outcomes among patients with STEMI with and without OHCA.

\section{METHODS \\ Data source}

This study was a retrospective analysis of prospectively collected data on consecutive patients with STEMI who presented to a Vancouver Coastal Health Authority (VCHA) hospital between 26 June 2007 and 31 March 2016. The VCHA STEMI database is a quality improvement tool that includes 13 participating hospitals in this region of which two are PCI-capable centres (and perform all the pPCIs in the region), and four are geographically close enough to refer patients with STEMI for pPCI. Patients at the other seven centres would not have been eligible for pPCI due to their distance from the PCI-capable centres.

\section{Patient population}

We identified 2929 consecutive patients with STEMI within VCHA who presented with an acute STEMI who survived to hospital admission between 26 June 2007 and 31 March 2016. Of these 2929 patients, 1020 patients underwent successful pPCI and were included in the analysis. We excluded patients who did not undergo a coronary angiogram $(n=130)$, those who did not undergo pPCI ( $\mathrm{n}=898 ; 206$ received fibrinolytic therapy) and patients with missing data (including OHCA status $(\mathrm{n}=996)$ or angiogram status unknown $(\mathrm{n}=14))$. OHCA status data were not systematically collected prior to 2012; therefore, there were only 36 patients between 2007 and 2012 whose OHCA status was known. Patients with STEMI were divided into those with $(n=91)$ and those without $(\mathrm{n}=929)$ preceding OHCA (figure 1).

\section{Definitions}

Patients with STEMI were identified after assessment by emergency medical services or by physicians in the emergency department. STEMI was defined using both clinical criteria (symptoms of ischaemia persisting for $20 \mathrm{~min}$ or longer) and ECG Criteria (ST elevation of $1 \mathrm{~mm}$ or more in any of the following areas: two or more anterior leads, two or more inferior leads, two or more posterior leads or presumed new left bundle branch block). A lesion was considered a culprit lesion if there was a fresh occlusion seen on angiography that resulted in subtotal or total occlusion. The occlusion was considered acute if there was a thrombus present at the site of the occlusion or if the guide wire was able to be passed through the occlusion easily. If there was no acute occlusion on angiography, it was at the angiographer's discretion to identify the lesion with the most severe reduction of lumen diameter along with corresponding localisation of ST-segment elevation on ECG. OHCA was defined as either having received attempts at defibrillation or chest compressions by lay bystanders or emergency medical services or being pulseless but not having received attempts at defibrillation or CPR. All data were collected in the VCHA STEMI database. Cardiogenic shock during the index hospitalisation was defined as a sustained episode of systolic blood pressure $<90 \mathrm{~mm} \mathrm{Hg}$ and/or cardiac index $<2.21 / \mathrm{min}$ / $\mathrm{m}^{2}$ determined to be secondary to cardiac dysfunction, and/or the requirement for inotropic or vasopressor agents or mechanical support (ventricular assist devices, intra-aortic balloon pump or extracorporeal circulation) to maintain perfusion above $90 \mathrm{~mm} \mathrm{Hg}$ and/or cardiac index $<2.21 / \mathrm{min} / \mathrm{m}^{2}$. Heart failure on presentation was defined as physician documentation or report of heart failure on presentation or any description of rales or pulmonary oedema on physical examination or chest 


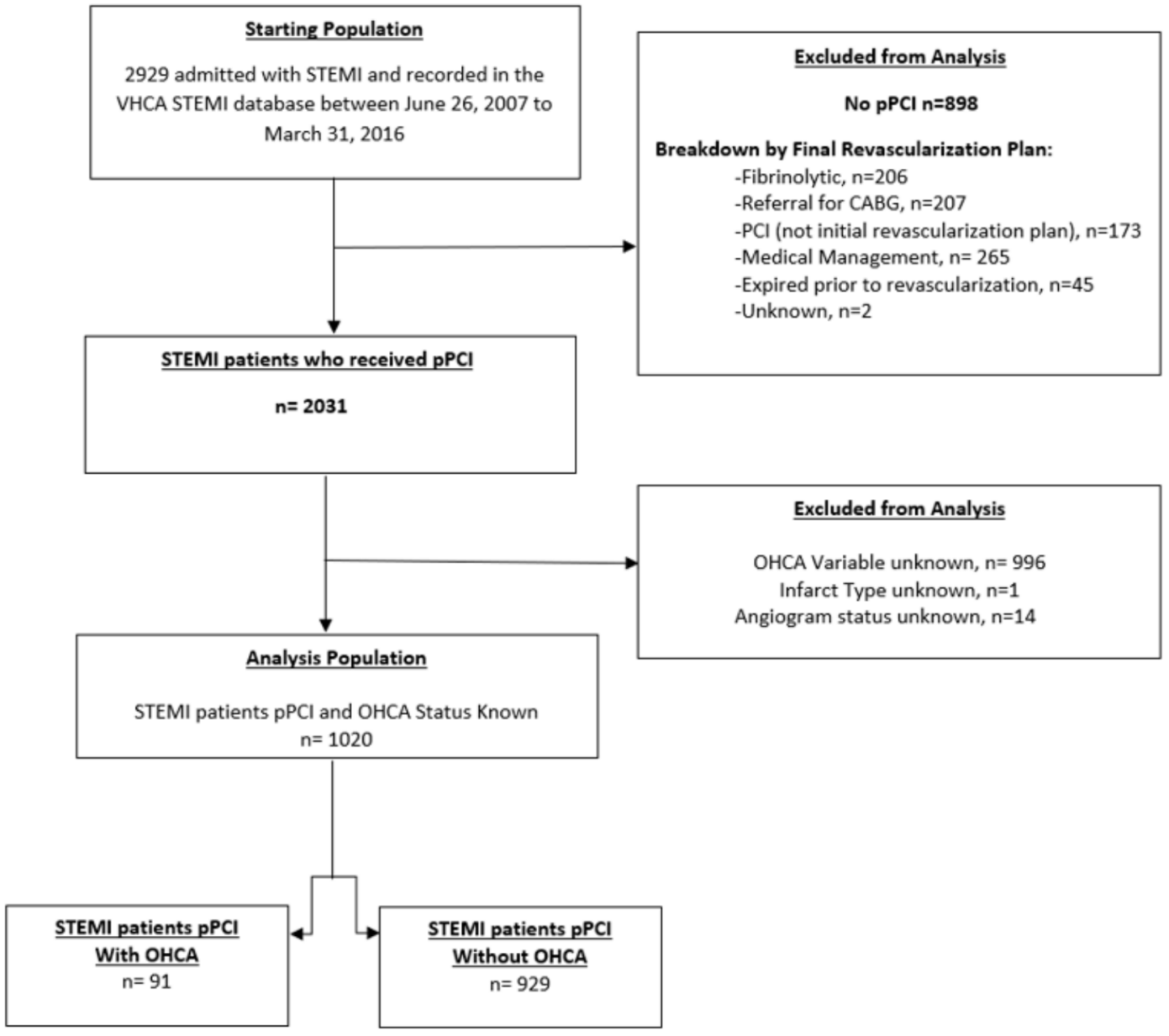

Figure 1 The cohort derivation from the initial study population, through exclusions, to the final study population. OHCA, out-of-hospital cardiac arrest; $\mathrm{PCl}$, percutaneous coronary intervention; $\mathrm{PPCl}$, primary percutaneous coronary intervention; STEMI, ST-segment elevation myocardial infarction; VHCA, Vancouver Coastal Health Authority; CABG, Coronary artery bypass grafting.

X-ray, jugular venous distention, shortness of breath, Killip class 2, 3 or 4 or presence of an S3. Major bleeding was defined as a suspected bleeding event during the index hospitalisation that was associated with significant blood loss (haematocrit drop of at least $10 \%$ and/ or a haemoglobin drop of at least $30 \mathrm{~g} / \mathrm{L}$ ), transfusion of whole blood or packed red blood cells, or use of a surgical or procedural intervention to stop bleeding.

\section{Statistical analysis}

Baseline patient demographics and clinical characteristics were summarised using means $(\mathrm{SD})$ or proportions as appropriate. Three separate comparisons were made: $\mathrm{LAD}$ versus non-LAD artery involvement within the OHCA group $\left(\mathrm{P}^{1}\right)$; LAD versus non-LAD artery involvement in the non-OHCA group $\left(\mathrm{P}^{2}\right)$; combined OHCA group versus the combined non-OHCA group $\left(\mathrm{P}^{3}\right)$. Differences were tested using the t-test, $\chi^{2}$ test or Fisher's exact test, as appropriate. In-hospital outcomes were reported across the groups $\left(\mathrm{P}^{1}-\mathrm{P}^{3}\right)$ and differences were tested using t-test for continuous variables, $\chi^{2}$ test for categorical variables or Fisher's exact test, as appropriate. Comparison of the effect of OHCA by LAD involvement $\left(\mathrm{p}_{\text {interaction }}\right)$ was based on linear regression or logistic regression, as appropriate. For all analyses, a p-value of $<0.05$ was considered significant.

Multivariable logistic regression analysis was used to examine the independent impact of ischaemic territory (LAD vs non-LAD) and OHCA on mortality. For left ventricular ejection fraction (LVEF), linear regression was used. We adjusted for age, diabetes, heart failure on presentation, cardiogenic shock on presentation and first medical contact to device time $>90 \mathrm{~min}$ (or $>120 \mathrm{~min}$ if first presented to a non-PCI capable hospital). These variables were selected a priori to determine the strength of the association between LAD territory and OHCA on mortality while adjusting for potential confounders. This regression model contained an interaction term between OHCA and ischaemic territory to examine the OHCA effect on mortality by ischaemic territory.

\section{RESULTS}

A total of 1020 patients with STEMI were identified. There were 91 patients with STEMI who had a preceding OHCA and 929 who had STEMI without OHCA. The 
Table 1 Clinical characteristics of study patients

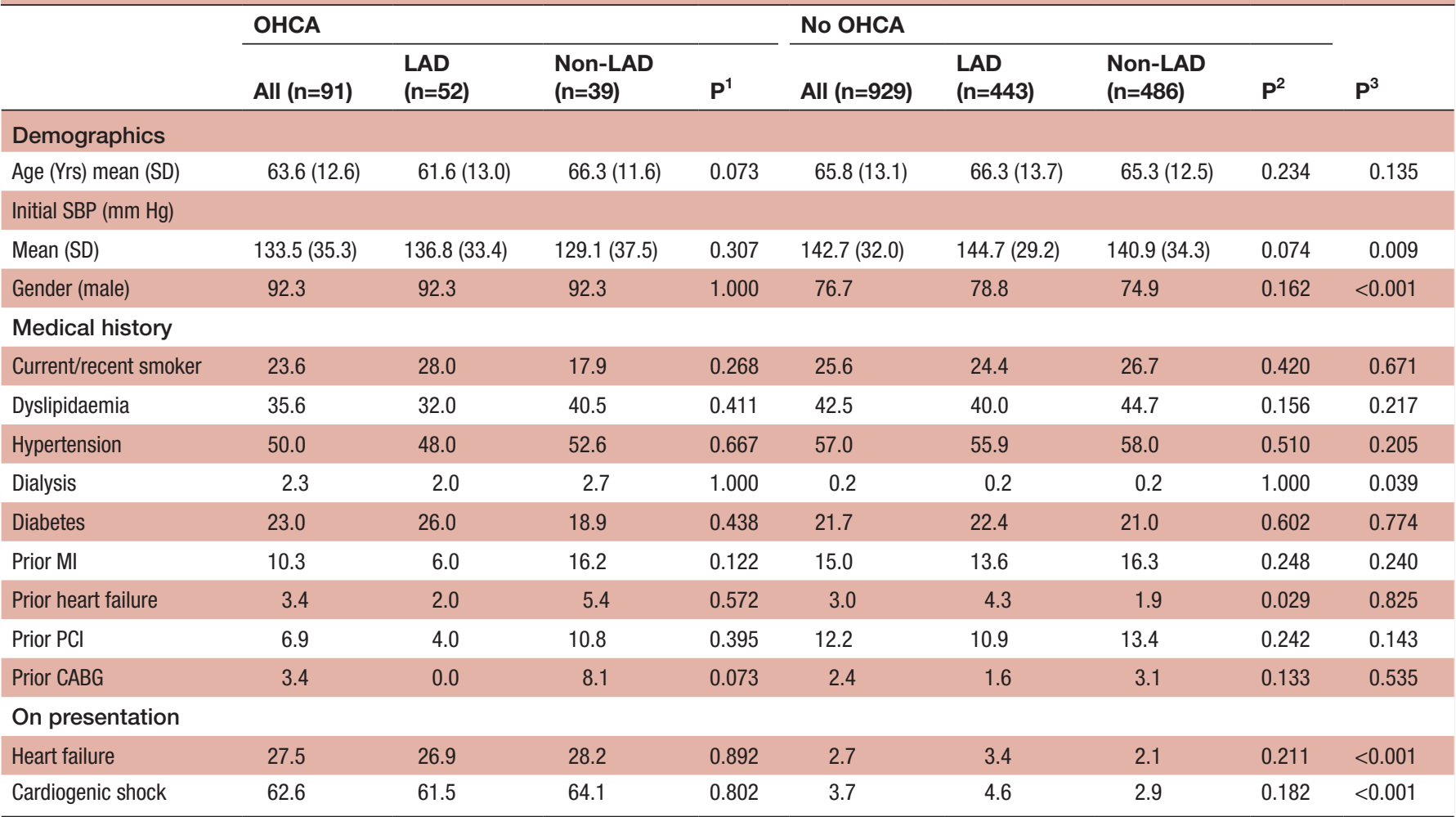

P1. Comparison between left anterior descending (LAD) and non-LAD within the out-of-hospital cardiac arrest (OHCA) group.

P2. Comparison between LAD and non-LAD within the non-OHCA group.

P3. Comparison between the combined OHCA group and the combined non-OHCA group.

Values are expressed as means or percentages.

CABG, Coronary artery bypass grafting; MI, myocardial infarction; PCI, percutaneous coronary intervention; SBP, systolic blood pressure.

culprit vessel and in-hospital outcomes for each patient was identified. Baseline characteristics of the cohort are described in table 1 . The mean age of patients with STEMI with OHCA was $63.6( \pm 12.6)$ years and $65.8( \pm 13.1)$ years among those without $(\mathrm{p}=0.135)$. A left anterior coronary artery (LAD) culprit was identified in $57.1 \%$ of patients with STEMI with OHCA and in $47.7 \%$ of patients with STEMI without OHCA (difference $=9.4 \%, 95 \%$ CI $-1.2 \%$ to $20.1 \%, \mathrm{p}=0.085$ ).

\section{Unadjusted analysis}

Patients with STEMI with OHCA had higher rates of in-hospital-cardiac arrest $(43.3 \%$ vs $8.3 \%, \mathrm{p}<0.001)$, heart failure $(50.5 \%$ vs $11.3 \%, \mathrm{p}<0.001)$, major bleeding $(22.0 \%$ vs $8.6 \%, \mathrm{p}<0.001)$, cardiogenic shock $(49.5 \%$ vs $5.7 \%, \mathrm{p}<0.001)$, mortality $(35.2 \%$ vs $3.3 \%, \mathrm{p}<0.001)$ and reduced LVEF ( $42.9 \%$ vs $47.3 \%, \mathrm{p}<0.001)$ compared with patients with STEMI without OHCA.

Among patients with STEMI with OHCA, the presence of an LAD culprit was not associated with increased risk of in-hospital mortality but was associated with a lower LVEF ( $40.6 \%$ vs $46.1 \%$, $\mathrm{p}=0.046$; figure 2 ). Conversely among patients with STEMI without OHCA, the presence of an LAD culprit vessel was associated with increased rates of heart failure $(18.1 \%$ vs $5.2 \%, \mathrm{p}<0.001)$, in-hospital cardiac arrest $(10.7 \%$ vs $6.2 \%, \mathrm{p}=0.014)$, cardiogenic shock $(8.4 \%$ vs $3.3 \%, \mathrm{p}<0.001)$, reduced LVEF
$(43.0 \%$ vs $51.2 \%, \mathrm{p}<0.001)$ and mortality $(5.2 \%$ vs $1.3 \%$, $\mathrm{p}=0.003$ ) compared with patients without an LAD culprit (figure 3).

Examining the outcomes of in-hospital cardiac arrest, major bleeding and LVEF revealed that the impact of OHCA was not significantly different by the presence of an $\mathrm{LAD}$ culprit vessel $\left(\mathrm{LAD}=40.4 \%\right.$, no $\mathrm{LAD}=47.4 \% \mathrm{p}_{\text {inter- }}$ action $=0.077, \mathrm{LAD}=25.0 \%$, no $\mathrm{LAD}=17.9 \% \mathrm{p}_{\text {interaction }}=0.405$, and $\mathrm{LAD}=40.6 \%$, no $\mathrm{LAD}=46.1 \% \mathrm{p}_{\text {interaction }}=0.232$, respectively). Conversely, the presence of an LAD culprit significantly altered the association between OHCA and congestive heart failure, cardiogenic shock and mortality $\left(\mathrm{LAD}=51.9 \%\right.$, no $\mathrm{LAD}=48.7 \% \mathrm{p}_{\text {interaction }}=0.009$, $\mathrm{LAD}=48.1 \%$, no $\mathrm{LAD}=51.3 \% \quad \mathrm{p}_{\text {interaction }}=0.033$ and $\mathrm{LAD}=30.8 \%$, no $\mathrm{LAD}=41.0 \% \mathrm{p}_{\text {interaction }}=0.007$, respectively).

\section{Adjusted analysis}

Logistic regression models demonstrated that the presence of OHCA was independently associated with mortality among patients with STEMI without (OR 7.75, $95 \%$ CI 2.03 to 29.68 ) but not with (OR 2.28, 95\% CI 0.76 to 6.87$)$ LAD involvement. However, the association between OHCA and mortality was not significantly different between each group $\left(p_{\text {interaction }}=0.112\right.$, figure 4$)$. The presence of an LAD culprit was independently associated with mortality among patients with STEMI without 


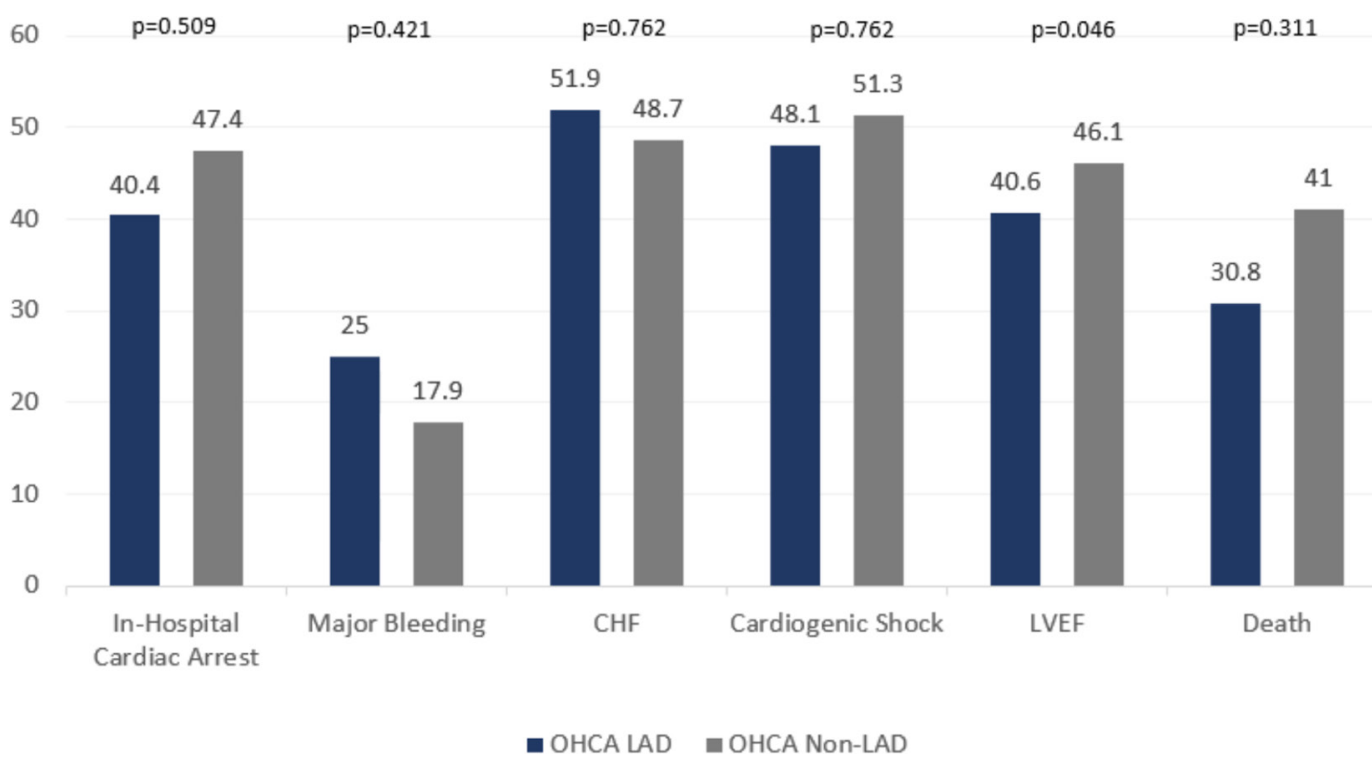

Figure 2 Unadjusted in-hospital outcomes in patients with ST-segment elevation myocardial infarction with out-of-hospital cardiac arrest (OHCA), by left anterior descending (LAD) involvement. CHF, congestive heart failure; LVEF, left ventricular ejection fraction.

OHCA (OR 2.79, 95\% CI 1.09 to 7.15 ) but not with OHCA (OR $0.82,95 \%$ CI 0.25 to 2.67 ).

Additionally, the presence of an LAD culprit was not independently associated with in-hospital cardiac arrest or major bleeding among patients with or without OHCA. In contrast, an LAD culprit was independently associated with heart failure and cardiogenic shock in patients without OHCA and not among patients with OHCA. An LAD culprit was independently associated with reduced ejection fraction in patients both with and without OHCA.

\section{DISCUSSION}

The current analysis sought to determine an association between an LAD culprit with in-hospital outcomes of patients with STEMI with and without OHCA. Our data confirmed that patients with STEMI with OHCA experienced worse in-hospital outcomes compared with those without. However, there was no independent association of an LAD culprit with mortality among patients with STEMI presenting with OHCA. Conversely, with the exception of in-hospital cardiac arrest, an LAD culprit retained an independent association with worse clinical

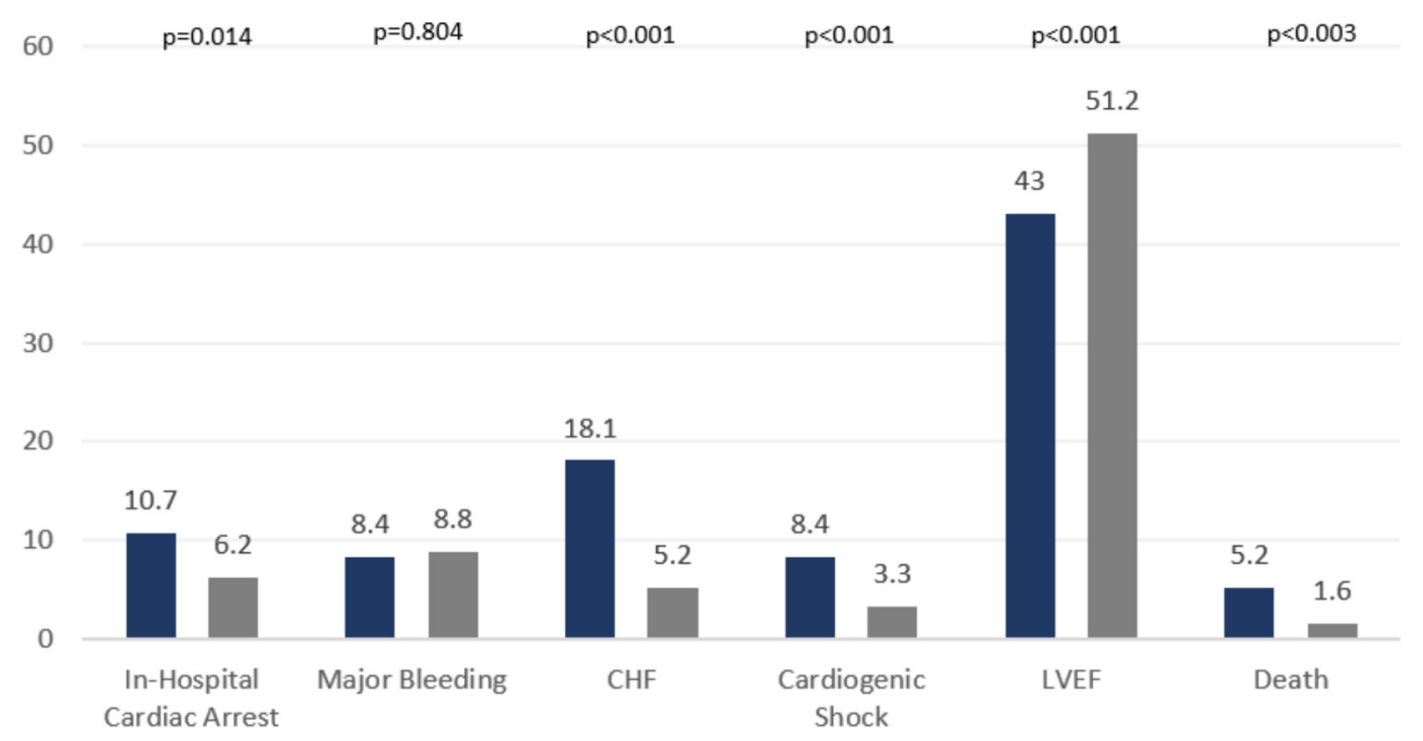

Without OHCA LAD Without OHCA Non-LAD

Figure 3 Unadjusted in-hospital outcomes in patients with ST-segment elevation myocardial infarction without out-of-hospital cardiac arrest (OHCA), by left anterior descending (LAD) involvement. CHF, congestive heart failure; LVEF, left ventricular ejection fraction. 


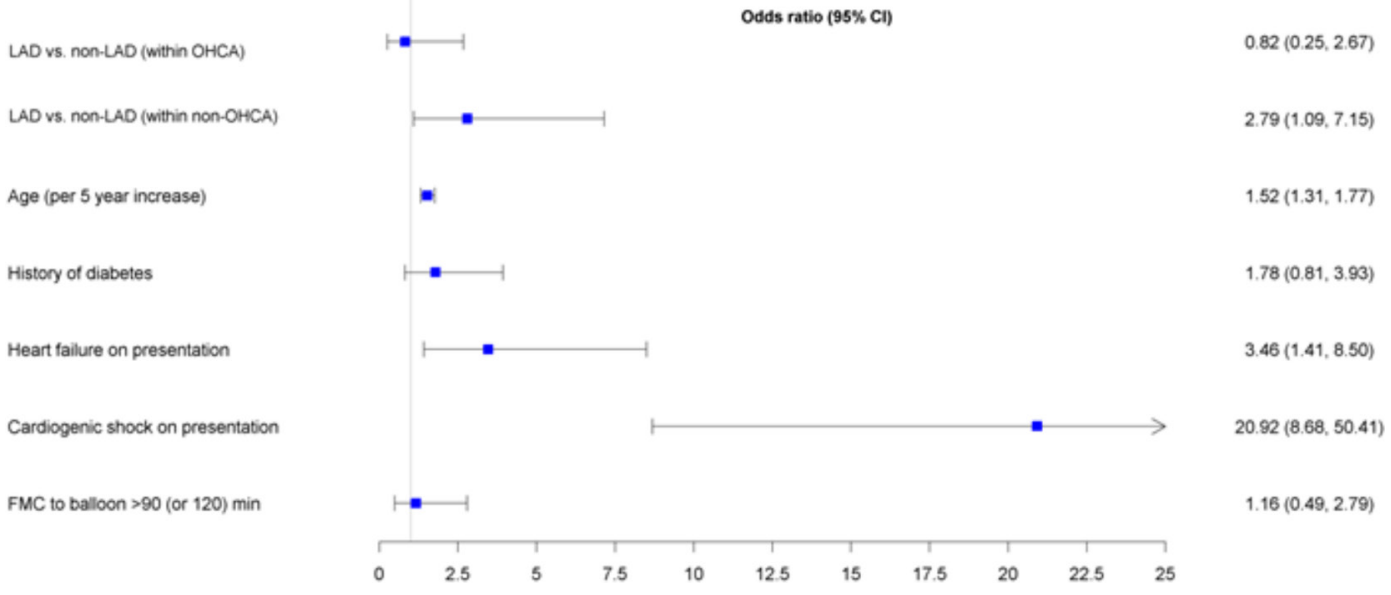

Figure 4 Logistic regression examining the impact of ischaemic territory (left anterior descending (LAD) vs non-LAD) and outof-hospital cardiac arrest (OHCA) on mortality. FMC, first medical contact.

outcomes among patients with STEMI without OHCA. ${ }^{20}$ Although a recent study has shown no clinical benefit of early angiography among OHCA survivors in the absence of acute ST elevation, ${ }^{21}$ non-randomised data have suggested favourable clinical outcomes with early angiography among OHCA with acute STEMI. ${ }^{22}$ However, prior to our study, little was known about whether specific angiographic features (and, in particular, LAD involvement) were independently associated with in-hospital mortality and other in-hospital outcomes among patients with STEMI with OHCA.

Although there is a suggestion of an increased incidence of left main coronary artery and multivessel disease involvement in patients with STEMI with OHCA compared with those without OHCA, ${ }^{10-12}$ the literature has yielded inconsistent signals regarding the presence of an LAD culprit and OHCA complicating STEMI $^{10-12} 1618$ nor the association between an LAD culprit and clinical outcomes among OHCA survivors with STEMI. ${ }^{10-12} 1819$ 23-25 Our study is one of the largest evaluating the association of $\mathrm{LAD}$ on outcomes among patients with STEMI with and without OHCA and extend on those from previous analyses. Our findings differ from previously described poor outcomes in non-OHCA presenting with acute anterior territory STEMI compared with non-anterior-related STEMI. ${ }^{11} 1314$ We hypothesised that there would be a higher incidence of LAD involvement among patients with STEMI with OHCA who survive to hospital admission, and that LAD involvement would confer worse in-hospital outcomes among patients with STEMI irrespective of OHCA status. Instead, we found similar rates of $\mathrm{LAD}$ involvement among patients with STEMI with and without OHCA. Moreover, our data also showed a differential association of LAD involvement with adverse in-hospital outcomes among patients with STEMI with and without OHCA, with a finding that LAD involvement was only associated with increased mortality among those patients without OHCA.

Our data would therefore suggest that the worse prognosis seen among patients with STEMI with OHCA is mainly driven by clinical characteristics other than the presence of high-risk angiographic anatomy, and that alternative mechanisms other than LAD involvement contribute to the higher mortality and worse hospital outcomes in patients with OHCA following successful reperfusion with pPCI. Several prehospital features have been associated with worse prognosis, including increased age, delayed CPR, initial unshockable rhythm, renal insufficiency, $>30 \mathrm{~min}$ to ROSC and reduced ejection fraction on hospital admission. ${ }^{15}{ }^{17}$ Indeed, integerbased risk scores based on clinical variables have been constructed to try and define which patients presenting with acute coronary syndromes are at increased risk of developing cardiac arrest. ${ }^{26}$

Clinicians treating patients with STEMI and OHCA should continue to use management strategies that emphasise early identification of patients with STEMI with OHCA and rapid reperfusion. However, clinicians should also focus on identifying and managing other concomitant high-risk features such as shock and neurological injury that drive poor outcomes in these patients given that approximately two-thirds of patients with OHCA who survive to hospital admission die from neurological injury, and the remaining one-third die from cardiogenic shock. ${ }^{27} 28$ As such, our study further highlights the complexity surrounding the prognostication following OHCA and underscores the need for further studies to delineate high-risk features in patients with STEMI with OHCA to help support clinical decision-making.

We acknowledge some limitations to our study. First, our data are observational and we cannot control for potential selection bias. In the present study, only patients who had ROSC and presented with ST-segment elevation were included in the analysis and we cannot exclude the possibility that the culprit artery of the successfully resuscitated patients could have varied significantly from those who did not have ROSC and never underwent angiography. In this way, it may be possible that there was either a higher incidence and/or a potential association of LAD with worse clinical outcomes among this cohort of 
patients who were not successfully resuscitated. However, we found no difference in the distribution of LAD involvement versus no LAD involvement among those patients who were sent for angiography. Second, our finding that there was no statistical association between OHCA and LAD may be a result of being underpowered with only 91 patients in this subgroup. There were relatively fewer patients with STEMI with OHCA than patients with STEMI without OHCA. However, there was a greater proportion of patients with LAD involvement in the OHCA group than in the non-OHCA group, though the lower number of patients with OHCA in this cohort may account for our inability to demonstrate a statistically significant difference in LAD involvement. Finally, the VCHA STEMI Registry does not contain detail regarding the precise location of the culprit lesion within the infarct-related artery; previous data have suggested that the presence of a proximal LAD culprit portends poorer outcomes among patients with STEMI. Therefore, it is possible that the presence of proximal versus nonproximal LAD disease may have impacted our results. ${ }^{28}$

\section{CONCLUSION}

Compared with patients with STEMI without OHCA, those with OHCA experienced worse in-hospital outcomes. While the presence of an LAD culprit was associated with worse outcomes among patients with STEMI without OHCA, this association was not seen among those with OHCA. LAD involvement was not independently associated with mortality in patients with OHCA but was seen in patients with STEMI without OHCA. These data suggest that there is a differential association of LAD culprit with poorer outcomes among patients with STEMI with and without OHCA and that other high-risk features may contribute to poor outcomes among patients with STEMI with OHCA. Further studies are needed to identify these high-risk features to support postarrest care clinical decision-making.

\section{Twitter Nima Moghaddam @nimamogh}

Contributors GW, CBF and MB conceived the paper and drafted the article. All authors contributed to the data analysis and interpretation. All authors were involved in the critical revision of the article and the final approval of the version to be published.

Funding The authors have not declared a specific grant for this research from any funding agency in the public, commercial or not-for-profit sectors.

Competing interests None declared.

Patient consent for publication Not required.

Ethics approval This study was approved by the University of British Columbia Clinical Research Ethics Board (H17-00375).

Provenance and peer review Not commissioned; externally peer reviewed.

Data availability statement All data relevant to the study are included in the article or uploaded as supplementary information. All data relevant to the study are included in the article or uploaded as supplementary information.

Open access This is an open access article distributed in accordance with the Creative Commons Attribution Non Commercial (CC BY-NC 4.0) license, which permits others to distribute, remix, adapt, build upon this work non-commercially, and license their derivative works on different terms, provided the original work is properly cited, appropriate credit is given, any changes made indicated, and the use is non-commercial. See: http://creativecommons.org/licenses/by-nc/4.0/.

ORCID iDs

Mia Bertic http://orcid.org/0000-0002-2796-0197

Nima Moghaddam http://orcid.org/0000-0003-0937-0641

Martha Mackay http://orcid.org/0000-0002-0290-9715

\section{REFERENCES}

1 McNally B, Stokes A, Crouch A, et al. Cares: cardiac arrest registry to enhance survival. Ann Emerg Med 2009;54:674-83.

2 Chan PS, McNally B, Tang F, et al. Recent trends in survival from out-of-hospital cardiac arrest in the United States. Circulation 2014;130:1876-82.

3 Mozaffarian D, Benjamin EJ, AS G, et al. Heart disease and stroke Statistics-2015 update. Circulation 2015;131:e29-322.

4 Nichol G, Thomas E, Callaway CW, et al. Regional variation in out-of-hospital cardiac arrest incidence and outcome. JAMA 2008;300:1423-31.

5 Dumas F, Cariou A, Manzo-Silberman S, et al. Immediate percutaneous coronary intervention is associated with better survival after out-of-hospital cardiac arrest. Circulation 2010;3:200-7.

6 Spaulding CM, Joly LM, Rosenberg A, et al. Immediate coronary angiography in survivors of out-of-hospital cardiac arrest. $N$ Engl $J$ Med 1997;336:1629-33.

7 Hosmane VR, Mustafa NG, Reddy VK, et al. Survival and neurologic recovery in patients with ST-segment elevation myocardial infarction resuscitated from cardiac arrest. J Am Coll Cardiol 2009;53:409-15.

8 Wong GC, van Diepen S, Ainsworth C, et al. Canadian cardiovascular Society/Canadian cardiovascular critical care Society/Canadian association of interventional cardiology position statement on the optimal care of the Postarrest patient. Can J Cardiol 2017;33:1-16.

9 Monsieurs K, Nolan JP, Bossaert LL, et al. European resuscitation Council guidelines for resuscitation 2015. resuscitation, European resuscitation Council guidelines for. Resuscitation 2015;95:1-80.

10 Zimmermann S, Flachskampf FA, Alff A, et al. Out-of-Hospital cardiac arrest and percutaneous coronary intervention for STelevation myocardial infarction: long-term survival and neurological outcome. Int J Cardiol 2013;166:236-41.

11 Stone PH, Raabe DS, Jaffe AS, et al. Prognostic significance of location and type of myocardial infarction: independent adverse outcome associated with anterior location. J Am Coll Cardiol 1988;11:453-63.

12 Elsman P, van't Hof AWJ, de Boer M-J, et al. Impact of infarct location on left ventricular ejection fraction after correction for enzymatic infarct size in acute myocardial infarction treated with primary coronary intervention. Am Heart J 2006;151:1239.e9-1239. e14.

13 Karha J, Murphy SA, Kirtane AJ, et al. Evaluation of the association of proximal coronary culprit artery lesion location with clinical outcomes in acute myocardial infarction. Am J Cardiol 2003:92:913-8.

14 Nienhuis MB, Ottervanger JP, Dambrink J-HE, et al. Comparative predictive value of infarct location, peak $\mathrm{CK}$, and ejection fraction after primary $\mathrm{PCl}$ for ST elevation myocardial infarction. Coron Artery Dis 2009;20:9-14.

15 McCullough PA, Prakash R, Tobin KJ, et al. Application of a cardiac arrest score in patients with sudden death and ST segment elevation for triage to angiography and intervention. $J$ Interv Cardiol 2002;15:257-61.

16 Gorjup V, Radsel P, Kocjancic ST, et al. Acute ST-elevation myocardial infarction after successful cardiopulmonary resuscitation. Resuscitation 2007:72:379-85.

17 Kroupa J, Knot J, Ulman J, et al. Characteristics and survival determinants in patients after out-of-hospital cardiac arrest in the era of 24/7 coronary intervention facilities. Heart Lung Circ 2017;26:799-807.

18 Siudak Z, Birkemeyer R, Dziewierz A, et al. Out-of-Hospital cardiac arrest in patients treated with primary PCI for STEMI. long-term follow up data from EUROTRANSFER registry. Resuscitation 2012;83:303-6.

19 Velders MA, van Boven N, Boden $\mathrm{H}$, et al. Association between angiographic culprit lesion and out-of-hospital cardiac arrest in ST-elevation myocardial infarction patients. Resuscitation 2013;84:1530-5.

20 Bertic M, Wong G, Fordyce C, et al. Association of left anterior coronary artery involvement on clinical outcomes among STEMI 
patients presenting with and without out-of-hospital cardiac arrest. Can J Cardiol 2017;33:S131-2.

21 Lemkes JS, Janssens GN, van der Hoeven NW, et al. Coronary angiography after cardiac arrest without ST-segment elevation. $N$ Engl J Med 2019;380:1397-407.

22 Camuglia AC, Randhawa VK, Lavi S, et al. Cardiac catheterization is associated with superior outcomes for survivors of out of hospital cardiac arrest: review and meta-analysis. Resuscitation 2014:85:1533-40.

23 Gheeraert PJ, Henriques JP, De Buyzere ML, et al. Out-ofHospital ventricular fibrillation in patients with acute myocardial infarction: coronary angiographic determinants. J Am Coll Cardiol 2000;35:144-50.

24 Tofler GH, Stone PH, Muller JE, et al. Prognosis after cardiac arrest due to ventricular tachycardia or ventricular fibrillation associated with acute myocardial infarction (the MILIS study). multicenter investigation of the limitation of infarct size. Am J Cardiol 1987;60:755-61.

25 Kyriakidis M, Petropoulakis P, Antonopoulos A, et al. Early ventricular fibrillation in patients with acute myocardial infarction: correlation with coronary angiographic findings. Eur Heart J 1993;14:364-8.

26 Faxén J, Hall M, Gale CP, et al. A user-friendly risk-score for predicting in-hospital cardiac arrest among patients admitted with suspected non ST-elevation acute coronary syndrome - The SAFERscore. Resuscitation 2017:121:41-8.

27 Laver S, Farrow C, Turner D, et al. Mode of death after admission to an intensive care unit following cardiac arrest. Intensive Care Med 2004;30:2126-8.

28 Califf RM, Armstrong PW, Carver JR, et al. Task force 5. Stratification of patients into high, medium and low risk subgroups for purposes of risk factor management. J Am Coll Cardiol 1996;27:1007-19. 